\title{
The Topology of Evolutionary Biology
}

Bärbel M. R. Stadler ${ }^{1}$ and Peter F. Stadler ${ }^{2,3,4}$

1 Max Planck Institute for Mathematics in the Sciences. Inselstrasse 22-26, D-04103 Leipzig, Germany stadler@mis.mpg.de, http://www.tbi.univie.ac.at/ baer/

2 Lehrstuhl für Bioinformatik, Institut für Informatik, Universität Leipzig, Kreuzstrasse 7b, D-04103 Leipzig, Germany.

peter.stadler@bioinf .uni-leipzig.de, http://www. bioinf .uni-leipzig.de/ studla/

${ }^{3}$ Insitut für Theoretische Chemie und Molekulare Strukturbiologie, Universität Wien, Währingerstrasse 17, A-1090 Wien, Austria

${ }^{4}$ Santa Fe Institute, Santa Fe, New Mexico

Summary. Central notions in evolutionary biology are intrinsically topological. This claim is maybe most obvious for the discontinuities associated with punctuated equilibria. Recently, a mathematical framework has been developed that derives the concepts of phenotypic characters and homology from the topological structure of the phenotype space. This structure in turn is determined by the genetic operators and their interplay with the properties of the genotype-phenotype map.

\section{Introduction}

Evolutionary change is the result of the spontaneous generation of genetic variation and the subsequent fixation of variants in the population through natural selection and/or genetic drift.

This is the basic assumption of the Neo-Darwinian model. Population genetics appears therefore as a natural framework for studying the evolution of phenotypic adaptation, the evolution of gene sequences, and the process of speciation, see e.g. [1,2]. Patterns of phenotypic evolution [3], however, such as the punctuated mode (the partially discontinuous nature) of evolutionary change [4], developmental constraints or constraints to variation [5,6], innovation [7], directionality in evolution, and phenotypic stability or homology $[8,9]$ are not adequately described by population genetics models.

The reason for this apparent discrepancy is that selection can determine the fate of a new phenotype only after it has been produced or "accessed" by means of variational mechanisms [?,10]. Phenotypes are not varied directly in a heritable fashion, but through genetic mutation and its consequences on development. The accessibility of a phenotype is therefore determined by the genotype-phenotype map (GP-map) which determines how phenotypes vary with genotypes [11-13].

The motivation for emphasizing the central role of the GP-map originates from studies in which RNA folding from sequences to secondary structures 
is used as a biophysically realistic, yet extremely simplified, toy-model of a genotype-phenotype map. Simulated populations of replicating and mutating sequences under selection exhibit many phenomena known from organismal evolution: neutral drift, punctuated change, plasticity, environmental and genetic canalization, and the emergence of modularity, see e.g. [13-18]. Laboratory experiments have also generated phenomena consistent with these patterns [19-21].

In this contribution we discuss in some detail the surprisingly far-reaching consequences of formalizing evolutionary theory starting from accessibility. This approach is to a large extent the consequence of the realization that there is a significant discrepancy between the mathematical setup of population genetics (the current implementation of the Neo-Darwinian model of evolution) and computational case studies of simple evolution processes mentioned above. Population genetics theory typically assumes that the set of possible phenotypes is organized into a highly symmetric and regular space equipped with a notion of distance; most conveniently, a Euclidean vector space [1]. Computational studies using an explicit genotype-phenotype model based on the RNA folding, however, suggest a quite different picture [13,17,22]: If phenotypes are organized according to genetic accessibility, the resulting space lacks a metric and is conveniently formalized by an unfamiliar structure that generalizes topological spaces [23-25].

\section{Genotype space}

The structure of genotype space is uniquely determined by the genetic operators at work: mutation, recombination, genome rearrangements, etc. In the case of point mutations and constant length genomes the situation is straight forward. Naturally, sequences that differ by a single mutation are neighbors in "sequence space" $[26,27]$. The sequence space can thus be represented as a graph, Fig. 1(a). The same is true for rearrangements.

In the RNA example of Fig. 2 below, a genotype is a sequence of nucleotides encoded by A, C, G, and $\mathrm{U}$ of a given length $n$. The genotype space consists of all $4^{n}$ sequences. Two sequences $x$ and $y$ are neighbors if they are different in just one position, i.e., if their Hamming distance is $d_{H}(x, y)=1$. The resulting graph, a generalized hypercube (Fig. 1) is highly symmetric.

The situation becomes more complicated, however, when recombination (crossover) is considered [28]. The analogue of the adjacency relation of the graph is the recombination set $\mathcal{R}(x, y)$, which is defined as the set of all (possible) recombinants of two parents $x$ and $y$. Recombination sets satisfy at least two axioms:

(X1) $\{x, y\} \in \mathcal{R}(x, y)$,

(X2) $\mathcal{R}(x, y)=\mathcal{R}(y, x)$.

Condition (X1) states that replication may occur without recombination, and (X2) means that the role of the two parents is interchangeable. Often a third 
condition

(X3) $\mathcal{R}(x, x)=\{x\}$

is assumed, which is, however, not satisfied by models of unequal crossover $[24,29]$. Functions $\mathcal{R}: X \times X \rightarrow \mathcal{P}(X)$ satisfying (X1), (X2), and (X3) were considered recently as transit functions [30] and as P-structures [31,32].

On the other hand, classical models of population genetics and quantitative genetics (tacitly) assume a euclidean vector space as the natural framework for studying the evolution of phenotypic adaptation, the evolution of gene sequences, and the process of speciation, see e.g. [1,2]. This begs the question whether there is a mathematical framework that contains graphs, recombination sets, and euclidean vector spaces as special cases. After all, accessibility in terms of the genetic operators is the common mechanism that "creates" the structure of genotype space.

We base our discussion on the notion of accessibility [13,17,23]. Let us write $x \curvearrowleft_{U} y$ to mean that $x$ is accessible from $y$ "at level $U$ ". Whether $x$ can be obtained from $y$ in practice depends on how long we are willing to wait. Alternatively, we might be interested whether we obtain $x$ from $y$ within a fixed time with a certain probability. The symbol $\curvearrowleft_{U}$ emphasizes that we consider accessibility w.r.t. a user-defined criterion.

The relation $x \curvearrowleft_{U} y$ is represented in an equivalent way by the set $U=\left\{(x, y) \mid x \curvearrowleft_{U} y\right\}$. Then $U[x]=\left\{y \mid x \curvearrowleft_{U} y\right\}$ can be interpreted as the $U$-neighborhood of $x$ : it is the set of all $y$ that have access to $x$ at level $U$. Suppose we have two accessibility relations $\curvearrowleft_{U^{\prime}}$ and $\curvearrowleft_{U^{\prime \prime}}$. Then their composition, defined by $x\left(\curvearrowleft_{U^{\prime}} \circ \curvearrowleft_{U^{\prime \prime}}\right) y$ if there is a $z \in X$ such that $x \curvearrowleft_{U^{\prime}} z$ and $z \curvearrowleft_{U^{\prime}} y$, is again an accessibility relation at a weaker level. Note that we do not require that two relations $\curvearrowleft_{U^{\prime}}$ and $\curvearrowleft_{U^{\prime \prime}}$ are comparable. There is a natural partial order, however, which is determined by the inclusion relation of the associated sets $U^{\prime}$ and $U^{\prime \prime}$ respectively: If $U^{\prime} \subset U^{\prime \prime}$ then $\curvearrowleft_{U^{\prime}}$ is a more stringently defined accessibility relation than $\curvearrowleft_{U^{\prime \prime}}$

From the mathematical point of view it is natural to consider the collection $\mathcal{U}$ of all accessibility relations on a given genotype space. This construction follows the spirit of [23] with small technical differences. What are the natural properties $\mathcal{U}$ ? We propose:

(UO) $X \times X \in \mathcal{U}$ (ergodicity).

(U1') $U^{\prime}, U^{\prime \prime} \in \mathcal{U}$ implies $U^{\prime} \circ U^{\prime \prime} \in \mathcal{U}$.

(U1) $U \in \mathcal{U}$ and $U \subseteq U^{\prime}$ implies $U^{\prime} \in \mathcal{U}$.

(U2) $x \curvearrowleft_{U} x$ for all $x \in X$ and all $U \in \mathcal{U}$.

The ergodicity hypothesis says that at some level everything is accessible from everywhere, if we just wait long enough or if we are content with sufficiently small probabilities. Axiom (U1') simply allows us to "combine" accessibility relations to multi-step processes that define weaker accessibility relations, and (U2) states the trivial observation that every point is accessible from itself. Axiom (U1) allows us to construct a (weaker) accessibility relations from a given one by adding arbitrary pairs - after all, by axiom 
(U0) arbitrary pairs are accessible at some level! If we assume that $\mathcal{U}$ is nonempty and axioms (U1) and (U2) are satisfied, then (UO) and (U1') hold as well. The set-system $\mathcal{U}$ on $X \times X$ is a generalized version of a uniformity. In the theory of uniform space [33] one usually assumes additional axioms, which, however, do not seem to be satisfied naturally in the case of genetic accessibility relations. These are:

(U3) $U, U^{\prime} \in \mathcal{U}$ implies $U \cap U^{\prime} \in \mathcal{U}$.

(U4) For each $U \in \mathcal{U}$ there is a $V \in \mathcal{U}$ with $V \circ V \subseteq U$.

(U5) $U \in \mathcal{U}$ implies $U^{-1}=\{(x, y) \mid(y, x) \in U\} \in \mathcal{U}$.

One speaks of pre-uniformities if (UO-U3) are satisfied, if (U4) also holds we have a quasi-uniformity, while for a uniformity (UO-U5) must hold. The (generalized) uniform structure $\mathcal{U}$ is associated with a generalized topology on $X$. In the following section we will briefly review this connection and the underlying mathematical framework.

\section{Generalized Topological and Uniform Spaces}

Textbooks on topology such as $[34,35]$ usually start by defining a topology on a set $X$ by means of a collection $\mathcal{O} \subseteq \mathcal{P}(X)$ of "open sets" (the power-set $\mathcal{P}(X)$ is the set of all subsets of $X)$. For our purposes it is more convenient to use the (equivalent) collection $\mathcal{C}=\{A \mid(X \backslash A) \in \mathcal{O}\}$ of closed sets as the primitive concept. A pair $(X, \mathcal{C})$ is a topological space if $\mathcal{C}$ satisfies the following four axioms ${ }^{1}$ :

(IO) $\emptyset \in \mathcal{C}$.

(I1) $X \in \mathcal{C}$.

(I2) If $A_{i} \in \mathcal{C}$ for all $i \in I$, then $\bigcap\left\{A_{i} \mid i \in I\right\} \in \mathcal{C}$.

(I3) If $A, B \in \mathcal{C}$ then $A \cup B \in \mathcal{C}$.

Here, $I$ is an arbitrary, possibly infinite, index set. In lattice theory more general so-called intersection structures are considered that fulfill only (I2), see e.g., [36]. The closure function $\mathrm{cl}: \mathcal{P}(X) \rightarrow \mathcal{P}(X)$ defined by

$$
\mathrm{cl}(A)=\bigcap\{B \in \mathcal{C} \mid A \subseteq B\}
$$

associates with each set $A \subseteq X$ its "closure" $\mathrm{cl}(A)$. The closure function cl has two important properties: (i) it is isotone, i.e., $A^{\prime} \subseteq A$ implies $\operatorname{cl}\left(A^{\prime}\right) \subseteq \operatorname{cl}(A)$ and (ii) it is idempotent, i.e., $\operatorname{cl}(\operatorname{cl}(A))=\mathrm{cl}(A)$. Given an isotone and idempotent closure function $\mathrm{cl}$, one recovers the associated intersection structure by setting $\mathcal{C}=\{C=\operatorname{cl}(A) \mid A \in \mathcal{P}(X)\}$. One can therefore just as well use the closure function $\mathrm{cl}$ to define a topology by means of the Kuratowski's axioms [37]:

$(K 0) \quad \mathrm{cl}(\emptyset)=\emptyset$.

\footnotetext{
${ }^{1}$ The corresponding axioms for open sets are obtained by exchanging unions and intersections.
} 


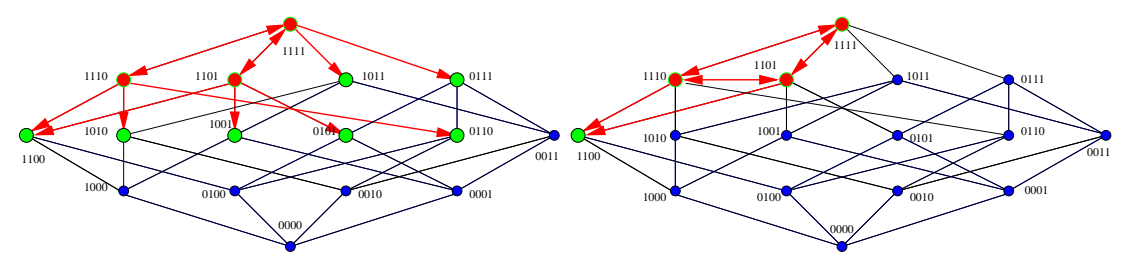

(a)

(b)

Fig. 1. Closure in Sequence Space.

A population $A=\{1111,1110,1101\}$ (red) and its closure $\mathrm{cl}(A)$ (in green) is shown for (a) point mutations, which give rise to the usual Hamming graph structure, and (b) 1-point recombination. In the latter case we show the embedding of $A$ and $\mathrm{cl}(A)$ in the Hamming graph since the resulting neighborhood space itself does not have a convenient representation. The homeomorphism of recombination spaces and Hamming graphs is discussed in detail in [28].

(K1) $A \subseteq B$ implies $\operatorname{cl}(A) \subseteq \mathrm{cl}(B)$.

(K2) $A \subseteq \mathrm{cl}(A)$.

(K3) $\operatorname{cl}(A \cup B) \subseteq \mathrm{cl}(A) \cup \mathrm{cl}(B)$ holds for all $A, B \subseteq X$.

(K4) $\operatorname{cl}(\mathrm{cl}(A))=\mathrm{cl}(A)$ holds for all $A \subseteq X$.

The dual of the closure function is the interior function int defined by $\operatorname{int}(A)=X \backslash \operatorname{cl}(X \backslash A)$ and hence $\operatorname{cl}(A)=X \backslash \operatorname{int}(X \backslash A)$. A set $N$ is a neighborhood of a point $x \in X$ if and only if $x \in \operatorname{int}(N)$. We write $\mathcal{N}(x)$ for the collection of all neighborhoods of $x$.

Kuratowski's closure axioms can be translated into the language of neighborhoods where $(\mathrm{Ni})$ is equivalent to $(\mathrm{Ki})$ above:

(N0) $X \in \mathcal{N}(x)$ for all $x \in X$.

(N1) $\quad N \in \mathcal{N}(x)$ and $N \subseteq N^{\prime}$ implies $N \in \mathcal{N}(x)$.

(N2) $\quad N \in \mathcal{N}(x)$ implies $x \in N$.

(N3) $N^{\prime}, N^{\prime \prime} \in \mathcal{N}(x)$ implies $N^{\prime} \cap N^{\prime \prime} \in \mathcal{N}(x)$

(N4) $\quad N \in \mathcal{N}(x)$ if and only if $\operatorname{int}(N) \in \mathcal{N}(x)$.

Generalized topological spaces have been used in various applied domains of computer science, such as digital image processing, information representation, the semantics of modal logic,handwriting recognition, and artificial chemistry, see e.g. [?,38-44] for a few examples.

A generalized neighborhood function $\mathcal{N}$ can be constructed from a generalized uniform structure $\mathcal{U}$ in a natural way. For each $x \in X$ and each $U \in \mathcal{U}$ we define the sets

$$
U[x]=\{y \in X \mid(x, y) \in U\} \quad \text { for each } U \in \mathcal{U} .
$$

and consider the collection

$$
\mathcal{U}[x]=\{N \mid \exists U \in \mathcal{U}: U[x] \subseteq N\}
$$

It is not hard to verify that $\mathcal{N}_{\mathcal{U}}: X \rightarrow \mathcal{P}(X), x \mapsto \mathcal{U}[x]$ is a neighborhood function on $X$. Conversely, given a neighborhood function $\mathcal{N}$ we may con- 
Table 1. Correspondence between generalized uniformities and generalized topologies.

\begin{tabular}{|llll|}
\hline & $(U 0-U 1)$ & $\leftrightarrow(N 0-N 1)$ isotone space \\
& $(U 0-U 2)$ & $\leftrightarrow(N 0-N 2)$ neighborhood space \\
preuniformity & $(U 0-U 3)$ & $\leftrightarrow(N 0-N 3)$ pretopology \\
quasiuniformity & $(U 0-U 4)$ & $\leftrightarrow(N 0-N 4)$ & topology \\
semiuniformity & $(U 0-U 3, U 5)$ & $\leftrightarrow$ & symmetric pretopology [45] \\
uniformity & $(U 0-U 5)$ & $\leftrightarrow$ & completely regular topology \\
\hline
\end{tabular}

struct a corresponding generalized uniformity as the collection $\mathcal{U}_{\mathcal{N}}$ of all sets $U$ that contain a set of the form

$$
U=\left\{(x, y) \mid x \in X \text { and } y \in N_{x} \text { for a fixed } N_{x} \in \mathcal{N}(x)\right\},
$$

$\mathcal{U}_{\mathcal{N}}$ is the generalized uniformization of the neighborhood system $\mathcal{N}$. The axioms for the generalized uniformities translate into properties of the resulting closure spaces as listed in Tab. 1. The correspondence of pre-uniformities and pretopologies is described in [23], for a proof that every topology is quasiuniformizable and for the characterization of uniformizable spaces see e.g. [33].

\section{Phenotype Space}

Accessibility at the phenotypic level is the crucial determinant for evolution since it is the phenotype that is subject to selection. We have seen in the previous section that the structure of genotype space is determined by physical processes, namely mutation and recombination, acting on genes. The phenotype, on the other hand, is therefore not modified directly, but indirectly through the modification of the genome from which it arises (We simplify here by disregarding e.g. epigenetic inheritance [46]). The accessibility relation at phenotypic level therefore has to be understood as a consequence of the interplay of genotypic accessibility and the GP-map.

The motivation for emphasizing the central role of the GPmap arose from a series of computer simulations in which fitness was modeled as a function of RNA secondary structures [13-18].

The RNA model, see Fig. 2, is a simplified, yet biophysically realistic, model for a GP-map that has the advantage that its predictions are amenable to experimental tests, see e.g. [47]. In nature, RNA molecules can act both as genotype (e.g. as genome of certain viruses) and as phenotype (the 3D structure can bind specifically to other molecules or even perform catalytic functions). These functions can then be "evaluated" by the environment e.g. in a replication experiment [48] or in a SELEX process [49]. RNA secondary structures are a convenient computational model because they can also be 


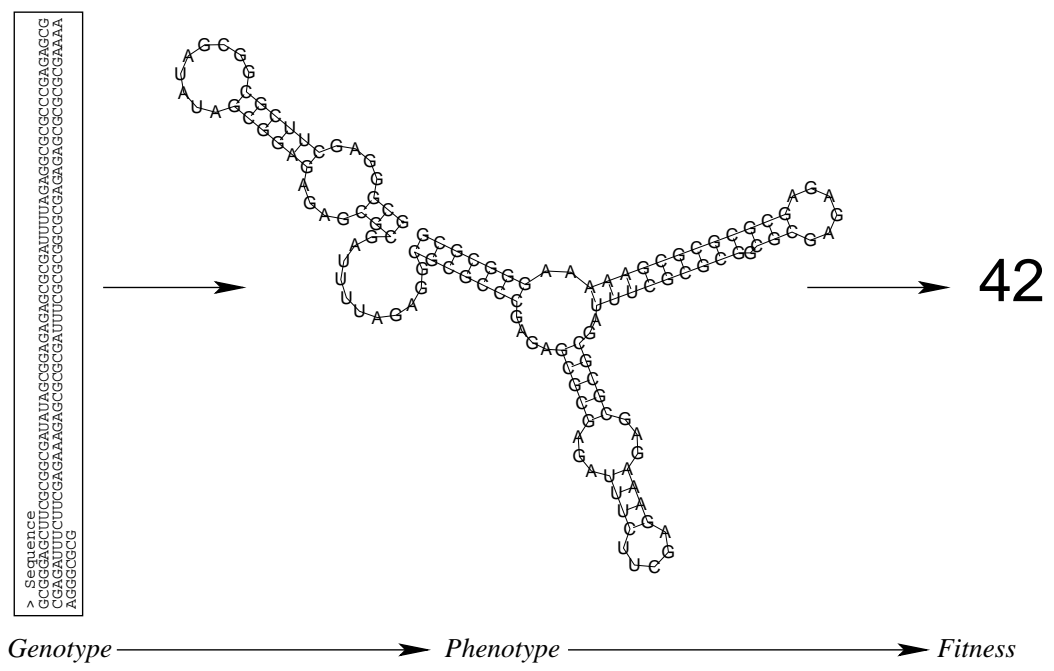

Fig. 2. An RNA molecule folds by first establishing the secondary structure, i.e., an outerplanar graph in which every nucleotide (letter) is connected to its sequence neighbors and to at most one other nucleotide with which it forms hydrogen bonds. The three-dimensional structure of the molecule is formed only in a second step, [52]. The main part of the energy of structure formation can be explained in terms of the base pair stacking at the secondary structure level [53]. The secondary structure of an RNA molecule is therefore a useful model for a biophysically realistic GP-map which serves as the basis for fitness landscapes that are obtained by assigning a fitness value to each secondary structure graph.

computed efficiently given only the sequence information by means of a dynamic programming algorithm $[50,51]$.

A generic feature of the GP-map at least of biopolymers is redundancy, i.e., there are many more sequences than structures (at a resolution of practical interest). Computational studies for both RNA [15] and protein [54] show quite convincingly that the set $\varphi^{-1}(\alpha)$ of sequences that fold into a common phenotype $\alpha$ forms a connected network in sequence space [15].

In practice, any useful notion of nearness will have to correlate with the likelihood of a transition from one phenotype $\beta$ to a "neighboring" one $\alpha$. For concreteness, let us consider a finite pretopological space, i.e., a directed graph, that might arise from a particular mutation operator. The possible mutants of a phenotype $\alpha$ then form the graph-theoretical boundary $\operatorname{bd} \varphi^{-1}(\alpha)$ of the neutral network of $\alpha$. The set $\varphi^{-1}(\beta) \cap \operatorname{bd} \varphi^{-1}(\alpha)$ is the subset of all these mutants that have phenotype $\beta$. Since the neutral networks are - at least in the RNA and protein case - approximately homogeneous we can estimate the probability of reaching $\beta$ from $\alpha$ as 


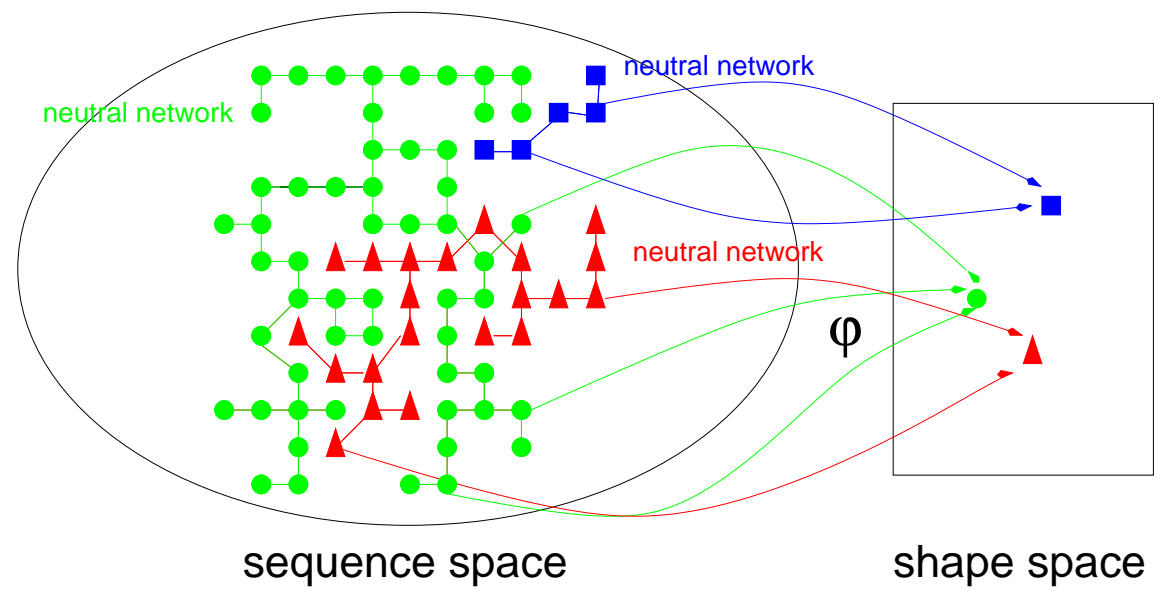

Fig. 3. Schematic representation of the neutral networks arising in RNA and protein folding.

$$
\chi(\beta \leftarrow \alpha)=\frac{\left|\varphi^{-1}(\beta) \cap \operatorname{bd} \varphi^{-1}(\alpha)\right|}{\left|\operatorname{bd} \varphi^{-1}(\alpha)\right|}
$$

where $|A|$ denotes the cardinality of the set $A$. Equ.(5) in turn defines a collection of accessibility relations $\curvearrowleft_{p}$ by setting

$$
\beta \curvearrowleft_{p} \alpha \Longleftrightarrow \chi(\beta \leftarrow \alpha) \geq p
$$

An approach to formulation accessibility in terms of so-called probabilistic closure spaces or, alternatively, fuzzy closure space is briefly discussed in [55].

Depending on the size of the underlying neutral nets, nearness between two phenotypes does not have to be symmetric. Consider a shape $\alpha$ with a very large, and a shape $\beta$ with a very small neutral network. It might be possible that almost all sequences in $\operatorname{bd} \varphi^{-1}(\beta)$ fold into shape $\alpha$, i.e., $\chi(\alpha \leftarrow \beta) \approx 1$, and $\alpha \curvearrowleft_{p} \beta$ for a large fixed value of $p$. On the other hand, we have $\chi(\beta \leftarrow \alpha) \approx 0$ because $\left|\varphi^{-1}(\beta)\right| \ll\left|\varphi^{-1}(\mathrm{bd} \alpha)\right|$, i.e, $\beta \bigwedge_{p} \alpha$. This effect can indeed be observed in the case of RNA secondary structures $[13,17]$. As a consequence, such asymmetries may introduce a directionality in evolution that is purely structural and hence independent of fitness.

\section{Continuity}

The notion of continuity lies at the heart of topological theory. Its importance is emphasized by a large number of equivalent definitions, see e.g. [56,57]. Let $(X, \mathrm{cl})$ and $(Y, \mathrm{cl})$ be two isotone spaces. Then $f: X \rightarrow Y$ is continuous if one (and hence all) of the following equivalent conditions holds: 
(i) $\mathrm{cl}\left(f^{-1}(B)\right) \subseteq f^{-1}(\mathrm{cl}(B))$ for all $B \in \mathcal{P}(Y)$.

(ii) $f^{-1}(\operatorname{int}(B)) \subseteq \operatorname{int}\left(f^{-1}(B)\right)$ for all $B \in \mathcal{P}(Y)$.

(iii) $B \in \mathcal{N}(f(x))$ implies $f^{-1}(B) \in \mathcal{N}(x)$ for all $x \in X$.

(iv) $f(\operatorname{cl}(A)) \subseteq \operatorname{cl}(f(A))$ for all $A \in \mathcal{P}(X)$.

We say that $f: X \rightarrow Y$ is continuous in $x$ if $B \in \mathcal{N}(f(x))$ implies $f^{-1}(B) \in$ $\mathcal{N}(x)$. Obviously, $f: X \rightarrow Y$ is continuous if it is continuous in each $x \in$ $X$. Continuity is preserved under function composition. If $f: X \rightarrow Y$ and $g: Y \rightarrow Z$ are continuous (in $x$ and $y=f(x)$ ), then $g \circ f: X \rightarrow Z$ is also continuous (in $x$ ).

The least stringent form of accessibility in phenotype space is defined by setting $\alpha \curvearrowleft \beta$ iff there is a sequence $y \in \varphi^{-1}(\beta)$ that has a potential offspring with phenotype $\alpha$, i.e.,

$$
\operatorname{cl}(\alpha)=\varphi\left(\operatorname{cl}\left(\varphi^{-1}\right)(\alpha)\right)
$$

In fact, this closure function on phenotype space is the most restrictive (finest) one with the property that $\varphi$ is continuous. We have argued in the previous section that in most cases a finer closure structure will reflect the practical accessibilities in phenotype space. It follows that the GP-map will in general not be continuous everywhere. We shall return to this topic in section 7 .

\section{Fitness and Fitness Landscapes}

Fitness landscapes were introduced in the 1930s by Sewall Wright $[58,59]$ as a means of visualizing evolutionary adaptation. In this picture a population moves uphill on a kind of "potential function" due to the combined effects of mutation and selection, Fig. 4. We have seen in the previous sections, however, that the horizontal - phenotypic - axis, in many cases, is not a real line but a neighborhood space. Alternatively, we may want to regard fitness landscapes as the graphs of functions from the genotype space with its neighborhood structure into the real numbers $\mathbb{R}$. This latter case is usually studied in simulations of molecular evolution.

Fitness and energy landscapes have become a unifying theme in fields as diverse as drug design, spin glass physics, molecular structure, protein folding, combinatorial optimization, and evolutionary theory, see e.g. [60] for a recent review. In each case, there is a function $f$, e.g., a molecular index, a Hamiltonian, a cost-function, or a fitness, that evaluates each member $x \in X$ of a (usually very large) configuration set $X$. These configurations can be (organic) molecules, spin configurations, conformations of polypeptide chain, tours of a TSP, or genotypes of living organisms.

Characteristic properties of fitness landscapes such as local minima and saddle points are inherently topological notions as well [61,62]. For instance, a point $x \in X$ is a local minimum of $f: X \rightarrow \mathbb{R}$ if there is a neighborhood $N \in \mathcal{N}(x)$ such that $f(y) \geq f(x)$ for all $y \in N$. It is instructive to consider 


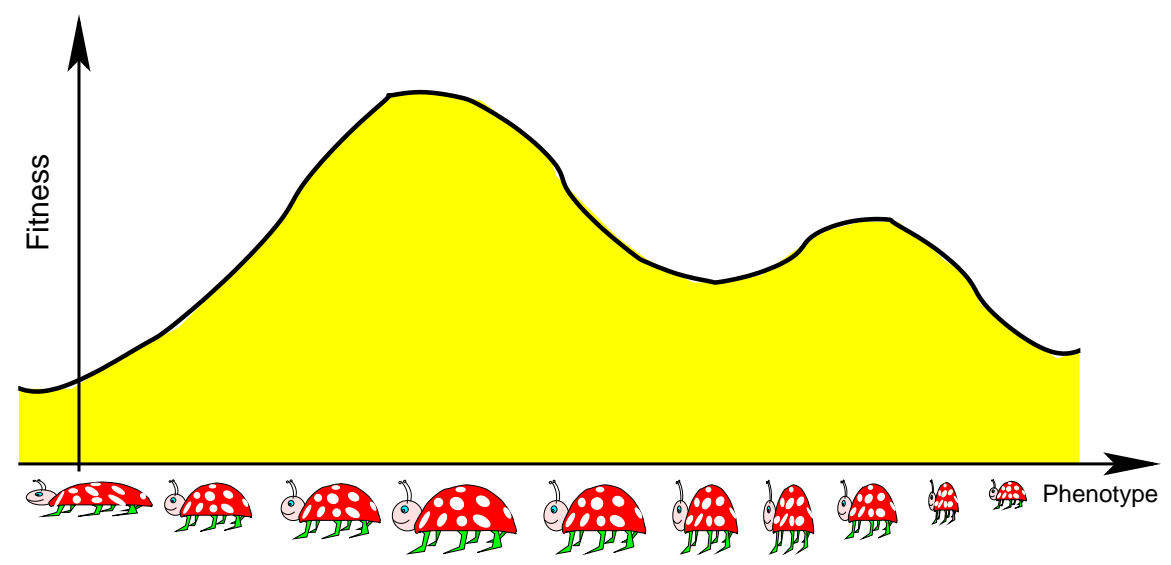

Fig. 4. Fitness landscapes assign fitness values to each (in this case) phenotypic state. The action of selection drives a population to local maxima of the fitness landscape.

also the following alternative definition: For each neighborhood $U \in \mathcal{N}(x)$ there is a neighborhood $N^{\prime} \subseteq U$ such that $f(x) \leq f(y)$ for all $y \in N^{\prime}$. Of course, in pretopological spaces both versions are equivalent: If $f(y) \geq$ $f(x)$ for all $y$ in a neighborhood $N$ then $N^{\prime}=U \cap N$ is another, smaller, neighborhood of $x$ which obviously satisfies $f(x) \leq f(y)$ for all $x \in N^{\prime}$. In general neighborhood spaces, however, the second definition is strictly stronger because the intersection $U \cap N$ of two neighborhoods is in general not a neighborhood of $x$ any more. This example highlights the pitfalls that appear because of the generality of the framework: "obvious" equivalences of properties that are familiar from topology textbooks might no longer be valid.

Related approaches to defining saddle points are discussed in [25]. The topological approach to fitness landscapes is largely unexplored. It appears promising, however, because it provides a common framework for the discrete landscapes of combinatorial optimization and for the continuum models that are used in evolutionary computation [63] and the analysis of chemical potential energy surfaces $[64,65]$.

\section{Evolutionary Trajectories}

An evolutionary trajectory can be regarded as a function $f$ from the time axis into phenotype space, where $f(t)$ represents the phenotype (e.g., the dominating phenotype in a population) at time $t$. Computer simulations $[15$, 16] using the RNA model described in Fig. 2 reveal a pattern of periods of stasis with intermittent bursts of adaptive evolution that is reminiscent 

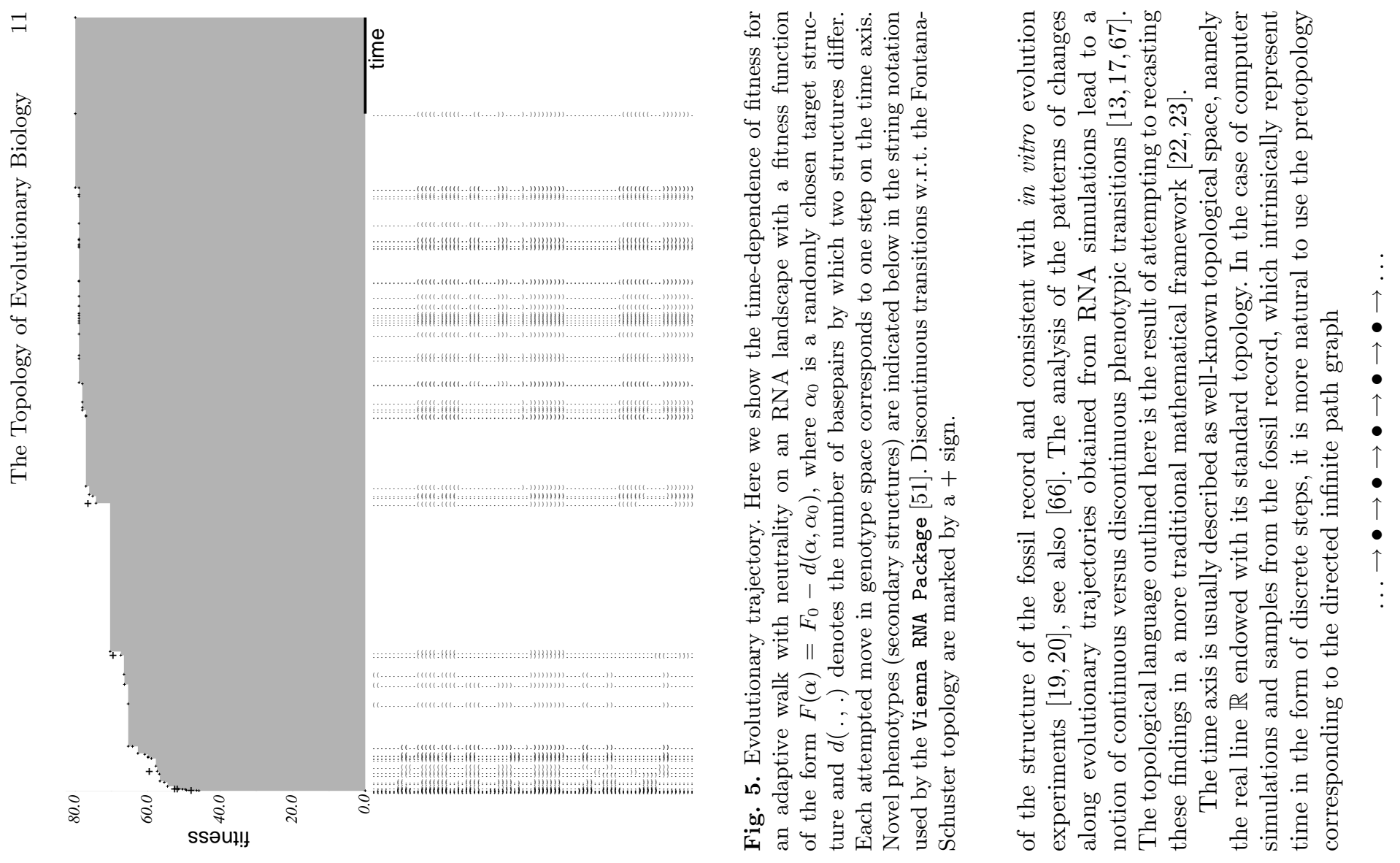
Let us write $\mathbb{T}$ for the generalized topological space that represents the time axis, and let $\xi: \mathbb{T} \rightarrow(X, \mathrm{cl})$ be a continuous function from the time axis into genotype space. Continuity means here simply that we assume that subsequently sampled genotypes are (easily) accessible from their predecessors. The composition $\varphi \circ \xi: \mathbb{T} \rightarrow(Y, \mathrm{cl})$ the describes the sequence of phenotypes as a function of time, i.e., $\varphi(\xi(t))$ is the phenotype at time $t$. Note that $\varphi \circ \xi$ cannot be guaranteed to be continuous everywhere, since the GP-map $\varphi$ will in general not be continuous.

The simplest approximation of an evolutionary process is the adaptive walk [68]. A population is represented as a single genotype which could be interpreted as the the consensus genotype. In each time step a mutant is explored and accepted if the fitness does not decrease. Thus the fitness $f=F \circ \varphi \circ \xi$ is monotonically increasing function of time. A typical trajectory is shown in Fig. 5. It does not differ qualitatively from the trajectories obtained in a more complex, population-based setting. In the RNA case, at least, there is a rather clear-cut distinction between "continuous transitions" (where $\varphi \circ \xi$ is locally continuous at a time $t_{0}$ where the phenotype changes) and discontinuous transitions. In particular, there is a close correspondence between continuity of a transition and the kind of structural changes that the RNA structure undergoes at the transition point. For more details we refer to $[13,17]$.

\section{Characters as Factors of Phenotype Space}

The correspondence of structural changes and transition type suggests that the topological language might be suitable to deal with a much more general question: Can one use the information about the evolutionary process that is represented by the topological structure of phenotype space to define a biologically meaningful character concept? In [23,69] we proposed that the most promising avenue is to start with Lewontin's notion of "quasiindependence" [70] which was introduced to clarify the mechanistic assumptions underlying the adaptationist research program.

Explaining a character state as an adaptation caused by natural selection requires the assumption that the character state can be produced by mutation without significantly affecting the functionality and/or structure of the rest of the body. This notion does not assume that genetic and mutational variation among characters is stochastically independent (it may in fact be strongly correlated). All that is assumed is that genetic variation can be produced at not too low rate so that natural selection can adjust one character without permanently altering other attributes of the phenotype. We can therefore interpret the notion of quasi-independence as a statement about the accessibility relations in the phenotypic configuration spaces, namely that the phenotype space can be represented as a product of generalized topological space, Fig. 6. 


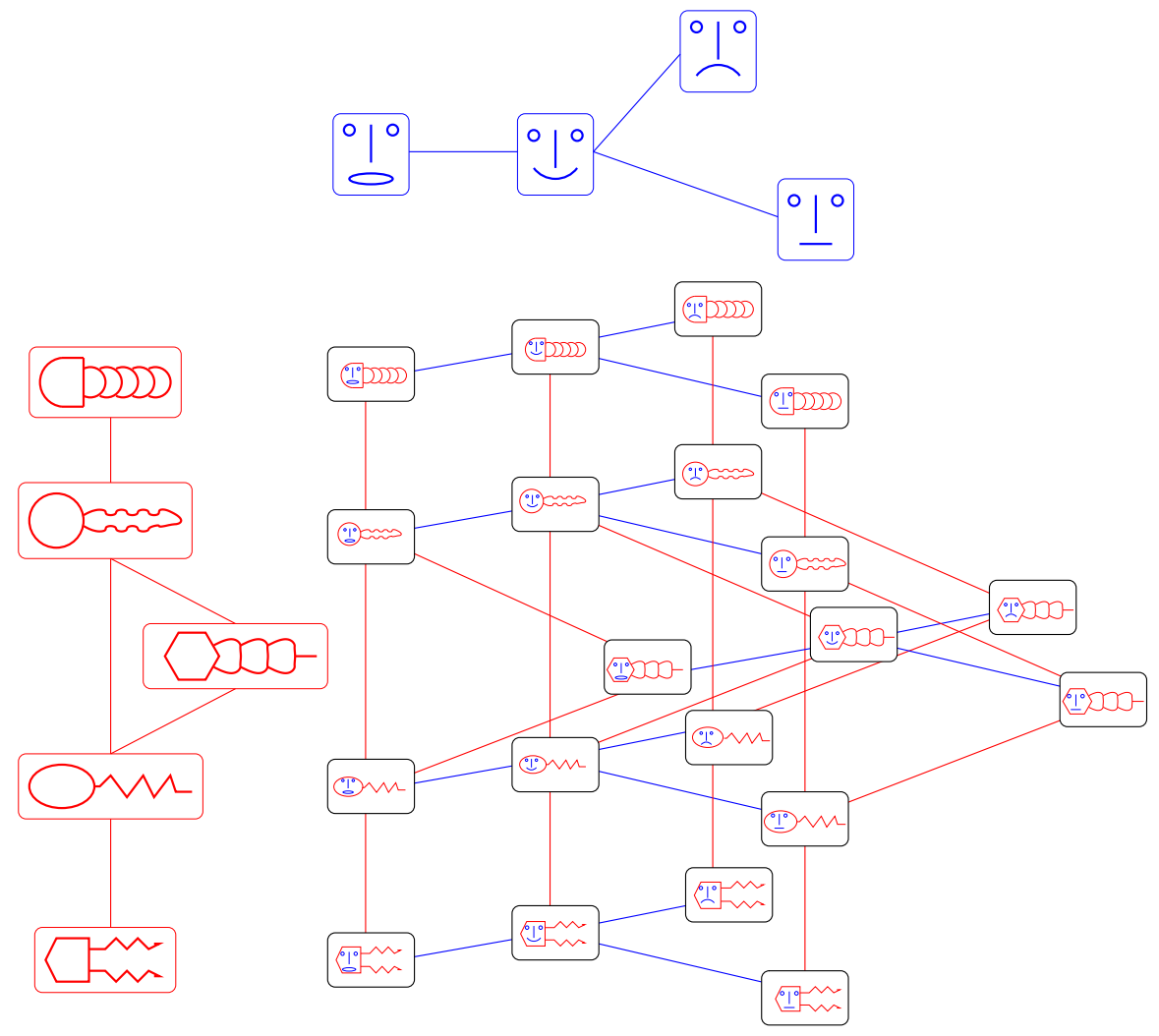

Fig. 6. Characters as factors of a graph. In this case we have two factors w.r.t. the Cartesian graph product, the "faces" and the "bodies" of the "animals". These are identified as the unique prime factors of their phenotype space graph. Note that while the representations of the "bodies" suggests "heads" and "tails" as finer subdivisions, these are not characters in our setting, because states of "head" and "tails" do not vary independently here.

In the following few paragraphs we outline very briefly how this approach works at the formal level. First we recall the definition the usual topological product and one of its interesting variants:

Let $(X, \mathcal{N})$ and $(Y, \mathcal{M})$ be two isotone space defined by their neighborhood functions. Then the (canonical) product space $(V, \mathcal{V})=(X, \mathcal{N}) \times(Y, \mathcal{M})$ is defined on the set $V=X \times Y$ and has the following neighborhood function $\mathcal{V}: U \in \mathcal{V}$ iff there is $N \in \mathcal{N}$ and $M \in \mathcal{M}$ such that $N \times M \subseteq U$. The space $(W, \mathcal{W})$ is the inductive product of $X$ and $Y$ if $W=X \times Y$ and $U \in \mathcal{W}$ iff there is $N \in \mathcal{N}$ and $M \in \mathcal{M}$ such that $(N \times\{y\}) \cup(\{x\} \times M) \subseteq U$. When the neighborhood function associated with each space is clear we write simply $V=X \times Y$ and $W=X \square Y$, respectively. The closure function of 
the inductive product can be characterized as follows. Consider two isotone spaces $\left(X, c_{X}\right)$ and $\left(Y, c_{Y}\right)$ and a subset $S \subseteq X \times Y$. The $(p, q) \in \operatorname{cl}(S)$ iff $p \in c_{X}(\{x \mid(x, q) \in S\})$ or $q \in c_{Y}(\{y \mid(p, y) \in S\})[45,17 . D .7]$, i.e.,

$$
\mathrm{cl}(S)=\left(c_{X}\left(S^{-1}[q]\right) \times\{q\}\right) \cup\left(\{p\} \times c_{Y}(S[p])\right) .
$$

In the case of connected graphs, i.e., finite pretopological spaces, the canonical and the inductive product reduce to the strong and Cartesian graph products, respectively; see [71] for their properties.

In the case of graphs the factorizations w.r.t. both the strong product and the Cartesian product are unique (up to automorphisms) [72,73]. Polynomial time algorithms for computing the prime factor decomposition of an undirected graph are known $[74,75]$. This is probably true also for finite neighborhood spaces although we do not yet have a formal proof for this claim. A "unique prime factor theorem" for closely related structures is given in $[76,77]$.

\section{Character Identity and Homology}

In $[23,69]$ we argue that quasi-independence is a local rather than a global notion. Local factorization means that the variational neighborhood of a phenotype can be described only in a neighborhood of a given phenotype by the combination of character states, i.e. the coordinates of "dimensions" or factors. The biological meaning of "locally factorizable" is that there are no variational limitations on realizing all possible combinations of adjacent character states. The range of phenotypes that can be described as a combination of states of a given set of character, on the other hand, may of course be limited. For instance, it may be possible to describe all fish species by a combination of a character states of the set of "fish characters", but there is no such set of characters which would describe the phenotypic disparity of all metazoans.

In mathematical terms the problem becomes to identify a factorizable subspace $Q$ of $(X, \mathrm{cl})$. In terms of graphs this means that we have to find induced subgraphs that are factorizable. Ideally, given a graph $G$ one would like to find maximal induced subgraphs that have non-trivial prime factor decompositions. Unfortunately, an efficient algorithms for this Induced Subgraph Factorization Problem is not known at present.

The original definition of homology by Owen identified two characters as homologous if they are "the same" in some unspecified way. The meaning of "sameness" was implicitly defined through the morphological criteria used to distinguish between superficial and essential similarity, i.e. between analogy and homology. This notion was re-interpreted by Darwin with reference to a common ancestor. It can be called the "historical homology concept" since it is defined solely on the basis of historical, genealogical relationships. It does 


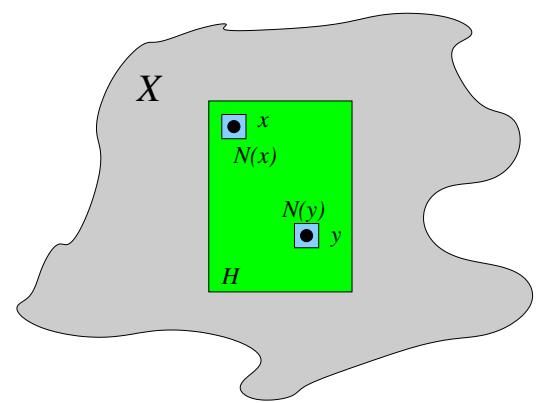

(a)

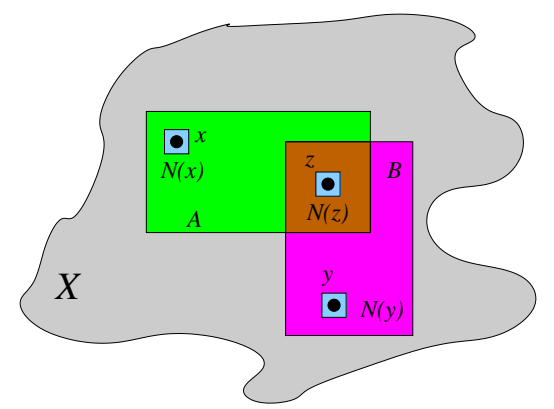

(b)

Fig. 7. A factorizable region $H$ established the identity of characters between two points $x, y \in \operatorname{int}(H)$. In a second step overlapping factorizable regions $A$ and $B$ can mediate character identity via points in their common interior $z \in \operatorname{int}(A) \cap \operatorname{int}(B)$.

not, however, clarify what "character identity" means [78]. Alternatively, one can define homologues as clusters of observable attributes that remain stable during adaptive evolution by natural selection. This "biological homology concept" $[8,9]$ is, in its definition, independent of relatedness by common of descent and thus has an unclear relationship to the historical homology concept.

Both homology concepts and their relationship can be accommodated in the topological theory of character identity [69]: In the previous section we have identified characters with local factors in phenotype space. The question of character identity hence can be rephrased as follows: Suppose we are given two points $x$ and $y$ and factorizations of their neighborhoods: can we identify factors of the neighborhoods that correspond to each other? The first step towards answering this question is to clarify the relationship between different subspaces. More formally, consider a neighborhood space $(X, \mathrm{cl})$ and let $\left(U, c_{U}\right)$ be a factorizable subspace, i.e., there are spaces $\left(U_{1}, c_{1}\right)$ and $\left(U_{2}, c_{2}\right)$ such that $\left(U, c_{U}\right)=\left(U_{1}, c_{1}\right) \times\left(U_{2}, c_{2}\right)$. Then every set of the form $A=A_{1} \times A_{2}$ with $A_{1} \subseteq U_{1}$ and $A_{2} \subseteq U_{2}$ is also a factorizable subspace [69]. In particular, every point $x \in \operatorname{int}(U)$ is locally factorizable, i.e., every neighborhood of $x$ contains a factorizable neighborhood. This property can then be used to establish the identity of characters (i.e., factors of local factorizations) at different points $x$ and $y$, provided there is a connected factorizable set $H$ such that $x, y \in \operatorname{int}(H)$, see Fig. 7a. The assumption of connectivity is crucial for the uniqueness of the factorization [71]. Overlapping factorizable regions may then be used to extend character identity to pairs of points that are not contained in a common factorizable region. The necessary condition is that there is a point $z \in \operatorname{int}(A) \cap \operatorname{int}(B)$ such that the local restrictions of the factors of $A$ and $B$ to sufficiently small neighborhoods of $z$ coincide. For the technical details we refer to [69]. 
The identity of variational characters is therefore well defined (via overlapping regional factorizations) and determines a class of (in most cases) variationally connected phenotypes sharing a certain factor. Phenotypes which share a certain factor/character can therefore evolve into each other without going through states where the character is not defined. The notion of character identity based on quasi-independence is thus fully consistent with the historical homology concept: Continuity of descent is sufficient to establish character identity. It is not a necessary conditions, however, because nothing in the general theory prevents two lineages from evolving phenotypes which have the same variational character - however unlikely this scenario might be.

\section{Concluding Remarks}

In this contribution we have discussed a mathematical framework that is capable of describing important aspects of macroevolution within the Darwinian framework. The language is general enough to be applicable in the same way to both the discrete setting of sequences and the continuum domain of population genetics; nevertheless it is powerful enough e.g. to construct a theory for continuity in evolution and to yield a meaningful notions of character and homology. Another interesting consequence to developmental evolution is discussed in [79].

We have focused on the formal aspects of this language rather than on real or potential applications. The reason is that the kind of variational data that would be necessary for a non-trivial example are not readily accessible and that a crucial technical step, namely the factorizable induced subgraph problem, remains yet to be solved.

\section{Acknowledgments}

The theory outlined in this contribution is joint work with Walter Fontana and Günter P. Wagner.

\section{References}

1. Futuyma, D.J.: Evolutionary Biology. Sinauer Associates, Sunderalnd, Massachusetts (1998)

2. Graur, D., Li, W.H.: Fundamentals of Molecular Evolution. Sinauer Associates, Sunderland, Massachusetts (2000)

3. Schlichting, C.D., Pigliucci, M.: Phenotypic Evolution: A Reaction Norm Perspective. Sinauer Associates, Inc., Sunderland, Massachusetts (1998)

4. Eldredge, N., Gould, S.J.: no title. In Schopf, T.J.M., ed.: Models in Paleobiology. Freeman, San Francisco (1972) 82-115 
5. Maynard-Smith, J., Burian, R., Kauffman, S.A., Alberch, P., Campbell, J., Goodwin, B., Lande, R., Raup, D., Wolpert, L.: Developmental constraints and evolution. Quart. Rev. Biol. 60 (1985) 265-287

6. Schwenk, K.: A utilitarian approach to evolutionary constraint. Zoology 98 (1995) 251-262

7. Müller, G.B., Wagner, G.P.: Novelty in evolution: Restructuring the concept. Annu. Rev. Ecol. Syst. 22 (1991) 229-256

8. Wagner, G.P.: The biological homology concept. Ann. Rev. Ecol. Syst. 20 (1989) 51-69

9. Wagner, G.P.: The origin of morphological characters and the biological basis of homology. Evolution 43 (1989) 1157-1171

10. Fontana, W., Buss, L.W.: "the arrival of the fittest":towards a theory of biological organisation. Bull. Math. Biol. 56 (1994) 1-64

11. Lewontin, R.C.: The Genetic Basis of Evolutionary Change. Columbia University Press, New York, New York (1974)

12. Wagner, G.P., Altenberg, L.: Complex adaptations and the evolution of evolvability. Evolution 50 (1996) 967-976

13. Fontana, W., Schuster, P.: Continuity in Evolution: On the Nature of Transitions. Science 280 (1998) 1451-1455

14. Fontana, W., Schnabl, W., Schuster, P.: Physical aspects of evolutionary optimization and adaption. Phys. Rev. A 40 (1989) 3301-3321

15. Schuster, P., Fontana, W., Stadler, P.F., Hofacker, I.L.: From sequences to shapes and back: A case study in RNA secondary structures. Proc. Roy. Soc. Lond. B 255 (1994) 279-284

16. Huynen, M.A., Stadler, P.F., Fontana, W.: Smoothness within ruggedness: The role of neutrality in adaptation. Proc. Natl. Acad. Sci. USA 93 (1996) 397-401

17. Fontana, W., Schuster, P.: Shaping Space: The Possible and the Attainable in RNA Genotype-Phenotype Mapping. J. Theor. Biol. 194 (1998) 491-515

18. Ancel, L., Fontana, W.: Plasticity, evolvability and modularity in RNA. J. Exp. Zoology (Molecular and Developmental Evolution) 288 (2000) 242-283

19. Spiegelman, S.: An approach to experimental analysis of precellular evolution. Quart. Rev. Biophys. 4 (1971) 213-253

20. Lenski, R.E., Travisano, M.: Dynamics of adaptation and diversification: A 10,000-generation experiment with bacterial populations. Proc. Natl. Acad. Sci. USA 91 (1994) 6808-6814

21. Szostak, J.W., Ellington, A.D.: In Vitro selection of functional RNA sequences. In Gesteland, R.F., Atkins, J.F., eds.: The RNA World. Cold Spring Harbor Laboratory Press, Plainview, NY (1993) 511-533

22. Cupal, J., Kopp, S., Stadler, P.F.: RNA shape space topology. Artificial Life 6 (2000) 3-23

23. Stadler, B.M.R., Stadler, P.F., Wagner, G.P., Fontana, W.: The topology of the possible: Formal spaces underlying patterns of evolutionary change. J. Theor. Biol. 213 (2001) 241-274

24. Stadler, B.M.R., Stadler, P.F., Shpak, M., Wagner, G.P.: Recombination spaces, metrics, and pretopologies. Z. Phys. Chem. 216 (2002) 217-234

25. Stadler, B.M.R., Stadler, P.F.: Generalized topological spaces in evolutionary theory and combinatorial chemistry. J. Chem. Inf. Comput. Sci. 42 (2002) $577-585$

26. Maynard-Smith, J.: Natural selection and the concept of a protein space. Nature 225 (1970) 563-564 
27. Eigen, M., Schuster, P.: The Hypercycle. Springer-Verlag, New York, Berlin (1979)

28. Gitchoff, P., Wagner, G.P.: Recombination induced hypergraphs: a new approach to mutation-recombination isomorphism. Complexity 2 (1996) 37-43

29. Shpak, M., Wagner, G.P.: Asymmetry of configuration space induced by unequal crossover: implications for a mathematical theory of evolutionary innovation. Artificial Life 6 (2000) 25-43

30. Changat, M., Klavžar, S., Mulder, H.M.: The all-path transit function of a graph. Czech. Math. J. 51 (2001) 439-448

31. Stadler, P.F., Wagner, G.P.: The algebraic theory of recombination spaces. Evol. Comp. 5 (1998) 241-275

32. Stadler, P.F., Seitz, R., Wagner, G.P.: Evolvability of complex characters: Population dependent Fourier decomposition of fitness landscapes over recombination spaces. Bull. Math. Biol. 62 (2000) 399-428

33. Page, W.: Topological Uniform Structures. Dover Publications, Inc., Mineola, NY (1994)

34. Gaal, S.A.: Point Set Topology. Academic Press, New York (1964)

35. Steen, L.A., Seebach, Jr., J.A.: Counterexamples in Topology. Holt, Rinehart \& Winston, New York (1970)

36. Davey, B.A., Priestley, H.A.: Introduction to Lattice and Order. Cambridge Univ. Press, Cambridge UK (1990)

37. Kuratowski, C.: Sur la notion de limite topologique d'ensembles. Ann. Soc. Polon. Math. 21 (1949) 219-225

38. Eckhardt, U., Latecki, L.: Digital topology. Technical Report 89, Hamburger Beitr. z. Angew. Math. A (1994)

39. Smyth, M.B.: Semi-metric, closure spaces and digital topology. Theor. Computer Sci. 151 (1995) 257-276

40. Pfaltz, J.: Closure lattices. Discrete Mathematics 154 (1996) 217-236

41. Galton, A.: Continuous motion in discrete space. In Cohn, A.G., Giunchiglia, F., Selman, B., eds.: Principles of Knowledge Representation and Reasoning: Proceedings of the Seventh International Conference (KR2000), San Francisco, CA, Morgan Kaufmann Publishers (2000) 26-37

42. Marchand-Maillet, S., Sharaiha, Y.M.: Discrete convexity, straightness, and the 16-neighborhood. Computer Vision \& Image Underst. 66 (1997) 316-329

43. Largeron, C., Bonnevay, S.: A pretopological approach for structural analysis. Information Sciences 144 (2002) 169-185

44. F. LeBourgeois, M. Bouayad, H.E.: Structure relation between classes for supervised learning using pretopology. In ???, ed.: Fifth International Conference on Document Analysis and Recognition. (1999) 33

45. Čech, E.: Topological Spaces. Wiley, London (1966)

46. Jablonka, E., Lamb, R.M.: Epigenetic Inheritance and Evolution. Oxford University Press, Oxford, UK (1995)

47. Schultes, E., Bartel, D.: One sequence, two ribozymes: Implications for the emergence of new ribozyme folds. Science 289 (2000) 448-452

48. Mills, D., Peterson, R., Spiegelman, S.: An extracellular darwinian experiment with a self-duplicating nucleic acid molecule. Proc. Nat. Acad. Sci. USA 58 (1967) 217

49. Klug, S.J., Famulok, M.: All you wanted to know about SELEX. Mol. Biol. Rep. 20 (1994) 97-107 
50. Zuker, M., Sankoff, D.: RNA secondary structures and their prediction. Bull. Math. Biol. 46 (1984) 591-621

51. Hofacker, I.L., Fontana, W., Stadler, P.F., Bonhoeffer, L.S., Tacker, M., Schuster, P.: Fast folding and comparison of RNA secondary structures. Monatsh. Chem. 125 (1994) 167-188

52. Shelton, V.M., Sosnick, T.R., Pan, T.: Applicability of urea in the thermodynamic analysis of secondary and tertiary RNA folding. Biochemistry 38 (1999) 16831-16839

53. Mathews, D., Sabina, J., Zucker, M., Turner, H.: Expanded sequence dependence of thermodynamic parameters provides robust prediction of RNA secondary structure. J. Mol. Biol. 288 (1999) 911-940

54. Babajide, A., Hofacker, I.L., Sippl, M.J., Stadler, P.F.: Neutral networks in protein space: A computational study based on knowledge-based potentials of mean force. Folding \& Design 2 (1997) 261-269

55. Stadler, P.F.: The genotype phenotype map. (2002) Konrad Lorenz Institute Workshop on Biological Information.

56. Hammer, P.C.: Extended topology: Continuity I. Portug. Math. 25 (1964) 77-93

57. Gniłka, S.: On continuity in extended topologies. Ann. Soc. Math. Pol., Ser. I, Commentat. Math. 37 (1997) 99-108

58. Wright, S.: The roles of mutation, inbreeding, crossbreeeding and selection in evolution. In Jones, D.F., ed.: Int. Proceedings of the Sixth International Congress on Genetics. Volume 1. (1932) 356-366

59. Wright, S.: "surfaces" of selective value. Proc. Nat. Acad. Sci. USA 58 (1967) $165-172$

60. Reidys, C.M., Stadler, P.F.: Combinatorial landscapes. SIAM Review 44 (2002) $3-54$

61. Flamm, C., Hofacker, I.L., Stadler, P.F., Wolfinger, M.T.: Barrier trees of degenerate landscapes. Z. Phys. Chem. 216 (2002) 155-173

62. Stadler, P.F.: Fitness landscapes. In Lässig, M., Valleriani, A., eds.: Biological Evolution and Statistical Physics, Berlin, Springer-Verlag (2002) 187-207

63. Rechenberg, I.: Evolutionstrategie. Frommann-Holzboog, Stuttgart (1973)

64. Heidrich, D., Kliesch, W., Quapp, W.: Properties of Chemically Interesting Potential Energy Surfaces. Volume 56 of Lecture Notes in Chemistry. SpringerVerlag, Berlin (1991)

65. Mezey, P.G.: Potential Energy Hypersurfaces. Elsevier, Amsterdam (1987)

66. Schuster, P.: Evolution in silico and in vitro: The rna model. Biol. Chem. 382 (2001) 1301-1314

67. Fontana, W.: Modelling "evo-devo" with RNA. BioEssays 24 (2002) 1164-1177

68. Kauffman, S.A.: The Origin of Order. Oxford University Press, New York, Oxford (1993)

69. Wagner, G., Stadler, P.F.: Quasi-independence, homology and the unity of type: A topological theory of characters. J. Theor. Biol. 220 (2003) 505-527

70. Lewontin, R.C.: Adaptation. Sci. Am. 239 (1978) 156-169

71. Imrich, W., Klavžar, S.: Product Graphs: Structure and Recognition. Wiley, New York (2000)

72. Dörfler, W., Imrich, W.: Über das starke Produkt von endlichen Graphen. Österreich. Akad. Wiss. Math.-Natur. Kl. S.-B. II 178 (1970) 247-262

73. McKenzie, R.: Cardinal multiplication of structures with a reflexive multiplication. Fund. Math. 70 (1971) 59-101 
74. Feigenbaum, J., Schäffer, A.A.: Finding the prime factors of strong direct products of graphs in polynomial time. Discr. Math. 109 (1992) 77-102

75. Imrich, W.: Factoring cardinal product graphs in polynomial time. Discr. Math. 192 (1998) 119-144

76. Lovász, L.: Operations with structures. Acta Math. Acad. Sci. Hung. 18 (1967) 321-328

77. Lovász, L.: Unique factorization in certain classes of structures. In: Mini-Conf. Univers. Algebra, Szeged 1971, Bolyai Janos Math. Soc. (1971) 24-25

78. Wagner, G.P.: Homology and the mechanisms of development. In Hall, B.K., ed.: Homology: The Hierarchical Basis of Comparative Biology. Academic Press, San Diego, California (1994) 273-299

79. Wagner, G.P.: What is the promise of developmental evolution? Part II: a causal explanation of evolutionary innovations may be impossible. J. Exp. Zool. (Mol. Dev. Evol.) 291 (2001) 305-309 